\title{
Corela
}

Cognition, représentation, langage

HS-30 | 2020

Phonétique, littérature et enseignement du FLE :

théories et recherches

\section{Appropriation multisensorielle du rythme du français : codage rythmique visuel d'extraits filmiques au sein d'un dispositif hybride}

Nadia Bacor

(2) OpenEdition

Journals

Édition électronique

URL : http://journals.openedition.org/corela/10837

DOI : 10.4000/corela.10837

ISSN : 1638-573X

Éditeur

Cercle linguistique du Centre et de l'Ouest - CerLICO

Référence électronique

Nadia Bacor, «Appropriation multisensorielle du rythme du français : codage rythmique visuel d'extraits filmiques au sein d'un dispositif hybride », Corela [En ligne], HS-30 | 2020, mis en ligne le 13 mai 2020, consulté le 22 mai 2020. URL : http://journals.openedition.org/corela/10837 ; DOI : https:// doi.org/10.4000/corela. 10837

Ce document a été généré automatiquement le 22 mai 2020

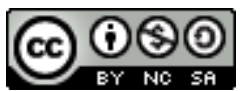

Corela - cognition, représentation, langage est mis à disposition selon les termes de la licence Creative Commons Attribution - Pas d'Utilisation Commerciale - Partage dans les Mêmes Conditions 4.0 International. 


\title{
Appropriation multisensorielle du rythme du français : codage rythmique visuel d'extraits filmiques au sein d'un dispositif hybride
}

\author{
Nadia Bacor
}

\section{Introduction : contextualisation et hypothèses de recherche}

L'enseignement/apprentissage du rythme du français est peu enseigné dans les classes de langue, soit par un manque de moyens matériels à disposition des enseignants de langue ou peu formés à la pédagogie de la prononciation à l'université (Lauret, 2007). Une brève histoire de l'enseignement de la prononciation (Wachs, 2011) montre que les méthodes issues du structuralisme avec la méthode audio-orale qui s'appuie sur le behaviorisme notamment, restent encore prégnantes de nos jours dans les manuels de Français Langue Étrangère (FLE) : a) avec la mise en relief des éléments segmentaux par la perception et $b$ ) la répétition des sons, au détriment de la prosodie de la langue dont fait partie le rythme. La méthode directe ensuite mise en avant repose sur la base d'un stimulus, d'une réponse puis d'un renforcement. Les exercices que l'on peut rencontrer sont donc des répétitions des composantes linguistiques qui visent la mémorisation de la prononciation, et ce de manière automatique. Or, avec l'avènement de l'Approche Communicative (1980) et de l'approche actionnelle (2000) mettant l'apprenant au cœur des apprentissages, on observe que de nouvelles pédagogies se mettent en place intégrant les différents aspects de la pratique langagière, comme les émotions. Ainsi, la composante prosodique du langage ressurgit dans l'apprentissage de la prononciation. 
2 Après les travaux de Delattre (1966), Rossi (1971; 1999), Di Cristo et al. (1993), Guimbretière (1994: 89) souligne que le rythme constitue l'un des paramètres les plus importants pour l'intercompréhension. Les proéminences influencent la compréhension lors de la situation de communication et donc interviennent dans le processus d'intelligibilité. En effet, l'accentuation possède diverses fonctions. Deux fonctions principales nous permettraient de différencier les systèmes rythmiques : une fonction distinctive et une fonction démarcative. La fonction distinctive permet de formaliser le changement de sens entre par exemple des phonèmes, des accentuations différentes ou bien des intonations différentes. La fonction démarcative, sert, quant à elle, à la formalisation d'un changement de sens aux frontières entre les groupes rythmiques par un mouvement mélodique (par exemple, intonation montante, descendante). Nous voyons ainsi l'importance du rythme dans la pratique langagière, mais s'agissant des enseignements, comment développer des méthodes pédagogiques permettant une appropriation du rythme par des moyens complémentaires à la perception et répétition de sons/mots/phrases ? La scénarisation pédagogique est dans cette perspective intéressante, et c'est l'objet de cet article.

Dans notre recherche, nous considérons le scénario cinématographique comme un genre littéraire permettant aux apprenants d'analyser les caractéristiques des personnages, leurs émotions, leurs histoires ancrées dans un schéma narratif dans un contexte socio-culturel donné. La construction du scénario cinématographique est essentielle notamment dans l'élaboration des dialogues proches de la langue parlée. En effet, d'après Vermeesch (2004), "l'importance du dialogue dans un scénario influence elle aussi son écriture car le dialogue est une sorte de compromis entre langue écrite et langue parlée ». Le texte du scénario « est lu et [...] doit plaire à ses lecteurs ", ce qui le rapproche du but d'un texte littéraire.

4 Dans cette recherche et à l'heure des apprentissages connectés, nous essayons de voir si l'alliance entre la composante visuelle par le scénario cinématographique et le codage rythmique constituent un atout pour l'appropriation visuelle du rythme du français.

5 Il est, de fait, essentiel de se demander comment développer la mémorisation sensorielle $d u$ rythme du français par un codage visuel du rythme d'extraits de films? Notre objectif est de voir en quoi la perception multisensorielle du rythme du français par la réalisation d'un codage visuel par couleurs et symboles d'extraits filmiques aiderait dans l'appropriation $d u$ rythme $d u$ français avec l'hypothèse selon laquelle ce codage rythmique visuel d'extraits de film permet un développement de la mémorisation sensorielle visuelle et auditive du rythme du français. Mais l'élaboration d'une telle scénarisation du rythme ne va pas de soi ni pour le chercheur ni pour le didacticien; c'est l'objet de notre développement.

6 Après avoir posé les fondements théoriques de l'enseignement/apprentissage du rythme du français en classes de langue pour des apprenants de FLE non-spécialistes de langues, l'approche méthodologique de notre dispositif pédagogique de scénarisation, illustré par des exemples de mise en pratique sera précisée, ainsi que le dispositif mis en place et issu d'une expérimentation dans le cadre d'une recherche-action (NarcyCombes, 2005). Le dispositif hybride (Nissen, 2019) retenu a pour objectif l'appropriation de la prononciation du français au niveau C1 du CECRL en contexte universitaire auprès de vingt-trois apprenants de FLE. 


\section{Fondements théoriques : lien entre rythme et stratégies d'apprentissage multisensorielles}

\section{1. Le rythme du français}

7 Les langues du monde sont classées actuellement d'après différentes catégories rythmiques. Le français, de façon schématisée, est une langue à isochronie syllabique, elle s'appuie sur un système rythmique se fondant sur une accentuation sur la dernière syllabe du groupe rythmique (Di Cristo, 2013). Sans revenir ici sur l'ensemble des fondements théoriques du rythme dont l'éclairage théorique depuis Delattre (1966), Wioland (1991) et Léon (2011) n'est plus à montrer, on sait à l'heure actuelle que l'appropriation du rythme reste un élément nécessaire pour l'intelligibilité et la compréhension mutuelle (Lauret, 2007 ; Guimbretière, 1994). Le rythme se définit selon Di Cristo et Hirst (1993) par «l'organisation temporelle des proéminences». Les proéminences seraient « les éléments mis en relief par des moyens tonals, intensifs et temporels » (Tortel, 2009:30) qui indique ainsi que «le rythme est un phénomène prosodique complexe qui agit comme principe générateur de structures ou de groupements plus ou moins récurrents, par la régularité ou l'alternance d'éléments saillants » (ibid. : 30).

Habituellement, trois paramètres prosodiques constituent le rythme : mélodie, durée et intensité ainsi que trois niveaux d'analyse du rythme qui s'offrent à nous : le niveau de la perception, de la production et le niveau linguistique, notamment lexical (Di Cristo, 2016). Il s'agit en effet de lier les "corrélats phoniques" du rythme aux "entités abstraites » au niveau linguistique. La perception du rythme au niveau physiologique se base sur la durée (des syllabes, des pauses, des proéminences), la mélodie (hauteur de l'accentuation lexicale, les intonations) et l'intensité (force de production d'un son associée à une énergie liée à la pression sous-glottique). Alors que sur le plan de la production, il s'agit d'un phénomène articulatoire avec la notion d'effort articulatoire pour la réalisation des proéminences (accentuation lexicale par exemple au niveau linguistique).

On ne peut plus sous-estimer la notion temporelle du rythme qui permet de dégager ces trois éléments fondamentaux du rythme: les proéminences, le groupe accentuel ou encore appelé groupe rythmique qui correspondent aux délimitations des frontières des mouvements mélodiques et d'intensité, parce qu'elles distinguent les unités de sens qu'abritent les groupes rythmiques (Morel et Danon-Boileau, 1998). Les variations temporelles et mélodiques, elles, sont caractérisées par des intonations montantes ou descendantes.

10 La notion de proéminence est primordiale dans cette recherche parce qu'il s'agit de mettre en relief ces éléments par un codage visuel. Ce sont les temps forts marqués par l'accentuation finale de la syllabe du groupe rythmique en français : l'apprenant qui y est exposé doit pouvoir extraire le sens de ce qui est dit. Le rythme serait ainsi une « organisation hiérarchique des niveaux de proéminence » (Di Cristo, 2004 ; 2016). Cette théorie faisait suite à la théorie de la Métrique du français (Liberman, 1975), repris ensuite par Hayes (1980) puis par Halle et Kenstowicz (1991) en tant qu'organisation hiérarchique de proéminences fondée sur la syllabe. Mais la définition évolue avec l'introduction de la notion temporelle. 


\section{2. La notion temporelle dans le rythme de la langue}

11 Di Cristo $(2004$; 2016) soulignera l'importance du «branchement binaire » avec la syllabe «s" (strong / forte) et la syllabe «w» (weak/faible), parce que les proéminences syllabiques vont définir le degré d'accentuation avec les règles rythmiques (Vogel, 1995) avec une alternance les temps forts et les temps faibles. Cette alternance est en relation avec le niveau lexical avec les groupes accentuels / rythmiques / de sens. La notion de hauteur permet de son côté de marquer le degré d'accentuation avec une représentation sur une ligne verticale avec l'une plus haute et l'autre plus basse.

Exemple : visuellement, la ligne haute représente l'accentuation de la dernière syllabe du groupe rythmique tandis que la ligne verticale basse représente les temps faibles. Le / symbolisant la démarcation grammaticale du groupe de sens correspondant au groupe rythmique. Nous avons fait les magasins, ce matin. Nous avons fait les magasins / ce matin.

$12 \mathrm{Au}$ niveau linguistique et notamment lexical, la notion de "groupe accentuel » ou encore de "groupe rythmique » en français, permet de définir la structure prosodique. Elle constitue un "assemblage hiérarchique de groupes accentuels", définis par des séquences de syllabes ne comprenant qu'un seul accent (une seule proéminence) placée en dernière position dans le groupe (Martin, $2012: 3$ ).

13 Di Cristo (1999 : 160) mentionne les travaux de Fónagy (1980) pour mettre en lumière la notion temporelle, la durée moyenne de l'intervalle inter-accentuel étant de l'ordre de $550 \mathrm{~ms}$ en français et le nombre de syllabes comprises entre deux accents successifs est majoritairement de trois à quatre syllabes : de 4 à 5 syllabes, selon Léon, (1992) dans la parole spontanée).

14 Ainsi, l'accentuation lexicale possède une hauteur plus haute, donc un ton haut (mélodie), une durée plus longue que les syllabes en position faible et une intensité plus forte. Ces paramètres sont importants pour notre recherche car nous allons mettre en place un symbole visuel pour représenter ces différents paramètres.

Des variations de durée peuvent être observées Léon (2011 : 139) : (dialectale, émotive, phonostylistique contrastive, allongement généralisé, textuelle contrôlée). Les groupes rythmiques (GR) sont aussi marqués par des pauses, des variations intra-individuelles comme la pause respiratoire, la pause grammaticale, la pause d'hésitation et la pause phonostylistique. Dans notre recherche, ils sont le reflet de la variation émotive en lien avec le codage rythmique des GR, de l'accentuation lexicale et des variations mélodiques: dans notre expérimentation, un codage des intonations montantes et descendantes qui influencent le rythme de la langue sont présentées en complément. Les paramètres de durée, d'intensité et de hauteur seront donc représentés par des symboles lisibles pour permettre un ancrage de l'information par le traitement cognitif sensoriel auditif et visuel.

Ces trois paramètres prosodiques rythmiques combinés (la mélodie, la durée et l'intensité) permettent à l'apprenant de percevoir et de produire les composants rythmiques par un biais linguistique. En comparant le système rythmique du français avec celui de l'anglais, par exemple, nous pouvons constater des différences 
intéressantes (Di Cristo, 2016) concernant la durée des syllabes accentuées et nonaccentuées ${ }^{1}$. "Le contraste entre les syllabes proéminentes et non-proéminentes est moins accusé en français que dans les langues anglo-saxonnes" (Di Cristo, 2016 : 66).Ces différences rythmiques ne sont pas sans incidence sur la perception et la production des apprenants ayant une langue première comme l'anglais. Selon la langue première des apprenants en classe de FLE, les difficultés récurrentes au niveau prosodique se situeraient, dans un premier temps, à l'écart entre le système métrique de leur langue première et leur langue-cible, ici le français. Un transfert ou une interférence rythmique peut bien entendu s'opérer entre la langue première et la langue seconde dans les recherches en acquisition de la langue (Kaglik et al., 2009). Comment acquérir la conscience rythmique notamment lorsque la langue première et la langue cible ne possèdent pas le même système rythmique?

De tels observables permettent aux apprenants de distinguer les divers niveaux dans le système rythmique $d u$ français, mais il faut matérialiser ces variations par une représentation visuelle de ces trois éléments qui favorise une mémorisation à long terme. Il s'agit donc d'allier perception auditive et visuelle pour matérialiser les phénomènes sonores. Concrètement, notre idée est de relier, pour ce faire, un symbole à chaque élément du rythme pour faciliter la compréhension des mécanismes du français parlé. Avant d'entrer plus en détail dans la méthodologie de ce codage visuel complexe, l'exploitation du scénario cinématographique en tant que support pédagogique privilégié pour le codage rythmique de la langue parlée du français mérite quelques précisions.

\section{3. Exploitation de scénarios cinématographiques : allier conjointement compréhension du scénario filmique et production orale}

18 Dans notre recherche, nous nous fondons sur l'appropriation visuelle d'extraits de scénarios de films en tant que texte littéraire. Le scénario, à la différence du script, « est construit sur un schéma narratif, comme un texte littéraire, auquel on ajoute une allusion continuelle au film à faire, quelques données techniques, un Temps, un Espace, un Mouvement et des Personnages qui ont un fonctionnement propre au cinéma, une disposition formelle particulière (en général la continuité dialoguée), une écriture propre, etc. » (VERMEESCH, 2004). Le scénario filmique existe en lui-même mais a pour objectif d'être représenté cinématographiquement (ibid.).

19 Le scénario cinématographique revêt ainsi un aspect littéraire de par sa nature narrative et par la construction du discours des personnages dans un contexte socioculturel. Bien que l'objectif de ce texte soit la représentation cinématographique, il n'en reste pas moins qu'il s'appuie sur la relation au temps, au contexte et aux dialogues. Lancien (1986) insiste déjà sur le fait qu'un film possède différentes composantes comme de la " langue écrite », de la « langue parlée » comme les dialogues par exemple, du « son » en relation avec des éléments visuels composés d'images.

Ces documents représentatifs des situations de communication et d'énonciation potentiellement rencontrées dans la vie quotidienne peuvent être utilisés dans le cadre de la classe de langue. Contrairement aux pièces de théâtres qui seraient généralement écrites pour être lues puis jouées sur scène, les scénarios des films et séries sont rédigés pour être mis en scène par des acteurs/comédiens. Ajoutons que les scénarios de films 
et séries reposent dans la majorité des cas sur la langue parlée spontanée de la vie quotidienne. Considérons que la langue utilisée dans le cadre des films est semispontanée (dans la mesure où les répliques ont été travaillées en amont de la mise en scène); le comédien ou l'acteur s'approprie par la suite un personnage et lui donne vie. Outre le potentiel esthétique de l'art cinématographique pour la mise en mouvance des émotions de l'apprenant, le jeu d'acteur correspond aux modes d'expressions actuels : "les signaux visuels, sensibles, "des organes sociaux" du corps sont constamment mêlés aux mots", disait Meschonnic (1982:654). L'art cinématographique va alors favoriser, voire déclencher l'émotion chez l'apprenant, les plans de vues sont étudiés par le réalisateur, situant le contexte de communication, le lieu, les locuteurs notamment leurs expressions verbales et non-verbales, les émotions. Le support (DVD notamment) permet également une souplesse dans l'utilisation en classe de langue par sa disponibilité et le choix des scènes correspondant aux thèmes du cours. Par ce travail sur les plans, il est possible d'obtenir des détails sur la situation d'énonciation avec notamment la possibilité d'un visionnage répété des scènes choisies. Le sens donné par l'image cinématographique rappelle Maury $(2012: 28)$ est «la prise de conscience par les apprenants de la fonction des techniques cinématographiques sur la construction du récit et donc du sens ».

21 Notre point de vue consiste à allier conjointement compréhension du scénario filmique et production orale pour déclencher un input langagier (Krashen, 1992) avec un travail sur les émotions des personnages (Damasio, 2010). De tels documents permettent de travailler sur les compétences interculturelles et langagières, grâce à la mise en place d'activités de compréhension orale du matériau filmique à travers le scénario. Medioni (2012) souligne à cet égard que c'est un facteur de motivation augmentant le "sentiment d'efficacité personnelle» (Bandura, 2003). Une des avantages de l'utilisation du matériau filmique est d'ailleurs la mise en place d'approches pédagogiques fondées sur l'apprendre en mettant en avant les stratégies expérientielles (issues de la théorie du « learning by doing » de Dewey, 1916).

Ces différentes situations d'énonciation que l'on retrouve dans les scènes de films (comprenant un énonciateur et un récepteur) sont socialement situées et authentiques, ce qui est essentiel en didactique des langues. Dans chaque scène nous avons un énonciateur et un récepteur avec un statut social, un lieu d'énonciation précis visible dans la scène du film (par le tournage dans le lieu d'énonciation et non par la reconstitution d'un décor sur scène), un message à adresser, avec l'utilisation d'un registre de langue spécifique à la communauté linguistique, à la catégorie socioprofessionnelle. Ces contextes de situations réelles chargées des sens socioculturels, avec la mise en relief du fonctionnement pragmatique développent des compétences périphériques à la prononciation (Myers, 2004).

Ce matériau filmique composé du scénario pédagogique retenu est couplé avec l'extrait de film correspondant, avec l'interprétation cinématographique du scénario. Nous avons ainsi trois types de supports : l'écrit, soit le scénario, les images du film ayant une fonction sémiotique forte permettant la compréhension avec la bande son (audio) ; la composante visuelle (vidéo) qui va donc se superposer à la troisième composante audio permettant un double codage de l'image et du son et favorisant ainsi un meilleur traitement du sens des dialogues avec les images. exposés, il convient de comprendre à présent en quoi le codage rythmique permettrait 
une meilleure appropriation du rythme par le développement des stratégies d'apprentissage multisensorielles du rythme.

\section{4. Des stratégies d'apprentissage multisensorielles du rythme du français}

D’après Atlan (1995 : 455), les stratégies d'apprentissage « peuvent être définies comme des connaissances métacognitives qui permettent de savoir quand et comment utiliser des processus et des données déjà à la disposition de l'apprenant». Dans cette recherche, nous nous focalisons sur les stratégies d'apprentissage cognitives, c'est-àdire les " opérations qui exigent l'analyse directe, la synthèse ou la transformation de la matière linguistique ». Les approches pédagogiques mises en place seraient donc " un arrangement systématique des événements d'apprentissage et/ou d'enseignement destiné à mettre en pratique les connaissances sur l'apprentissage de façon prévisible et efficace afin d'atteindre des objectifs d'apprentissage définis" (ibid.:457). Les stratégies d'apprentissages multisensorielles (visuel, auditive et kinesthésique) seraient des stratégies permettant une meilleure mémorisation et appropriation de la connaissance ${ }^{2}$. Ainsi, la mémoire à long terme est active lorsque les informations données ont été répétées plusieurs fois pour un traitement en profondeur (Eustache et Desgranges, 1999). L'idée est d'allier la mémorisation échoïque des sons et la mémorisation sensorielle pour une appropriation à long terme du rythme. Le traitement des informations sur le rythme aura, comme il a été dit précédemment, un impact sur la mémorisation à long terme. Allons plus loin en décrivant le dispositif mis en place.

\section{Méthodologie}

Dans notre expérience, nous nous appuierons sur une appropriation du rythme du français par la mémorisation multisensorielle issue d'un développement de stratégies d'apprentissage multisensorielles qui fait l'objet une recherche de thèse en Didactique des Langues en cours ${ }^{3}$. Notre article inclut l'un des aspects de la perception multisensorielle du rythme, à savoir sa perception visuelle par le développement d'un codage rythmique (Abercrombie, 1964 ; Anderson-Hsieh, 1992 ; Di Cristo, 1993 ; 1999 ; 2016) utilisant un système de couleurs et de symboles représentant ses différents composants; le marquage des proéminences (accentuation), les délimitations des proéminences et de la durée (groupes rythmiques) et les contours mélodiques (intonations montantes et descendantes) en mettant ainsi en exergue les trois paramètres prosodiques qui sont la mélodie, la durée et l'intensité (Di Cristo, 2016).

Cette représentation visuelle (Jacquinot, 1985 ; 2012) du rythme est très importante elle réside dans le développement de la mémorisation non seulement auditive (lors de la perception) mais également visuelle. Notre idée est de combiner deux modes de mémorisation simultanément pour ancrer l'information à long terme. On pose que cette mémorisation sensorielle visuelle (Gaonac'h et Cordier, 2010) permet notamment un développement des stratégies d'apprentissage auditives et visuelles. 


\section{1. La mise en place d'un dispositif hybride pour l'appropriation de la prononciation du français}

Notre recherche-action (en cours d'exploitation) s'appuie sur la mise en place d'un dispositif hybride de douze semaines comprenant vingt-quatre heures de formation, à raison de deux heures par semaine en présentiel et vingt minutes à distance par semaine avec la mise en place d'activités sur la plateforme LMS Moodle. Cette formation a pour objectif l'appropriation de la prononciation du français avec une approche multisensorielle fondée sur la mise en scène théâtrale d'extraits de films permettant un développement des stratégies d'apprentissage multisensorielles, justement pour une mémorisation multisensorielle du rythme (kinesthésique pour la mise en scène théâtrale, auditive pour l'extrait de film et visuelle pour le codage rythmique).

\section{2. Scénarisation du dispositif en présentiel}

Le dispositif est composé de douze séances en présentiel et douze sessions de vingt minutes à distance entre chaque séance en présentiel. Le dispositif a pour tâche finale la réalisation d'un vidéoguide sur un élément du patrimoine culturel immatériel francophone. Il est composé de six thèmes d'actualités en relation avec les thèmes du patrimoine culturel immatériel comme la gastronomie, l'espace, les nouvelles technologies, les langues, le climat et la consommation de biens culturels.

Dans cette contribution, nous nous focaliserons sur la séance 4 dédiée à l'accentuation des syllabes en fin de groupe rythmique avec pour thème l'exploration spatiale. Les séances 1 à 3 sont consacrées à la perception et la démarcation des groupes rythmiques. Les séances 4 à 7 sont focalisées sur l'accentuation, les séances 8 à 10 permettent d'acquérir les compétences sur la perception et la production des liaisons et des enchainements et enfin les séances 11 et 12 sont dédiées aux intonations montantes et descendantes en relation avec les émotions. Voici les thèmes des douze séances : 


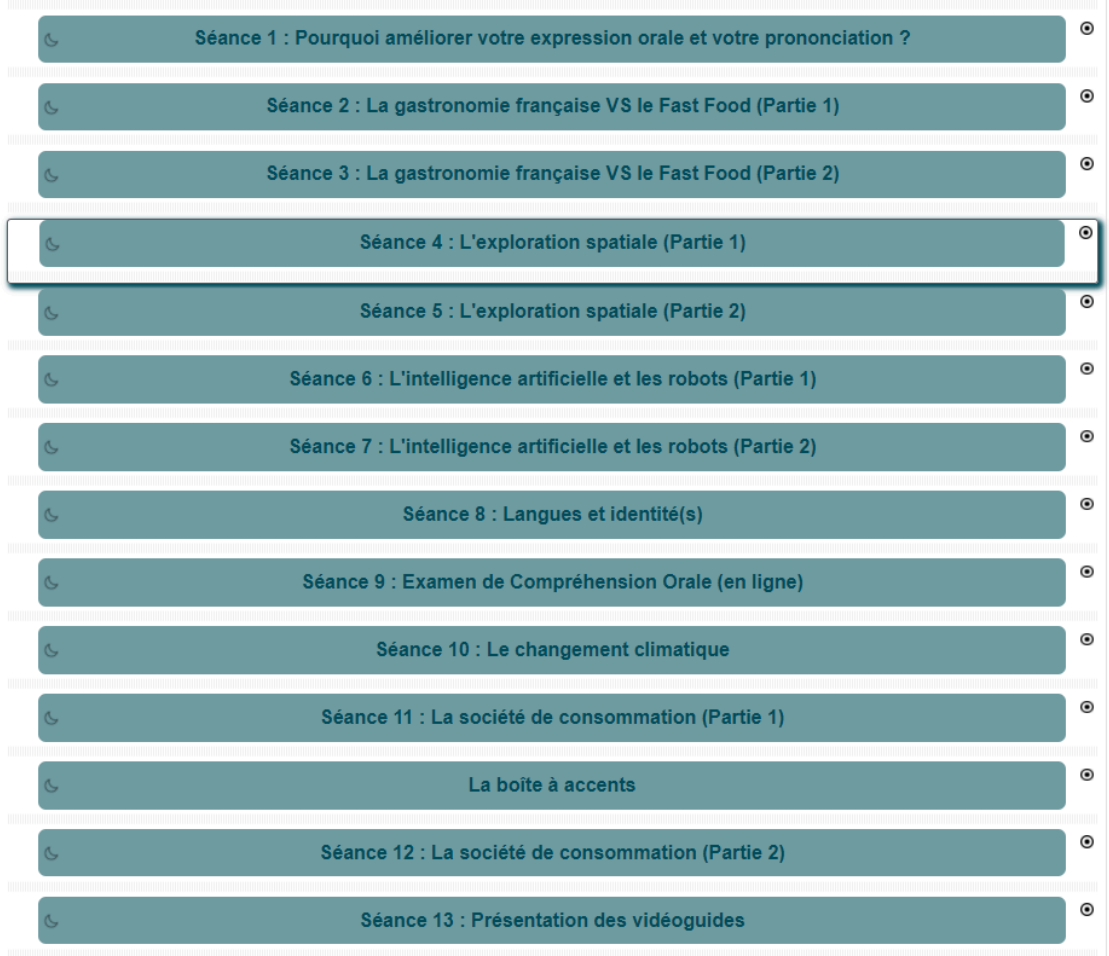

Figure 1 : scénarisation pédagogique du dispositif hybride étudié

(capture d'écran des douze séances à distance sur la plateforme Moodle)

31 Chaque séance est basée sur différentes étapes pour favoriser la perception et la production du rythme du français. L'objet est de mettre en évidence l'élaboration du scénario pédagogique :

\section{Scénarisation du dispositif}

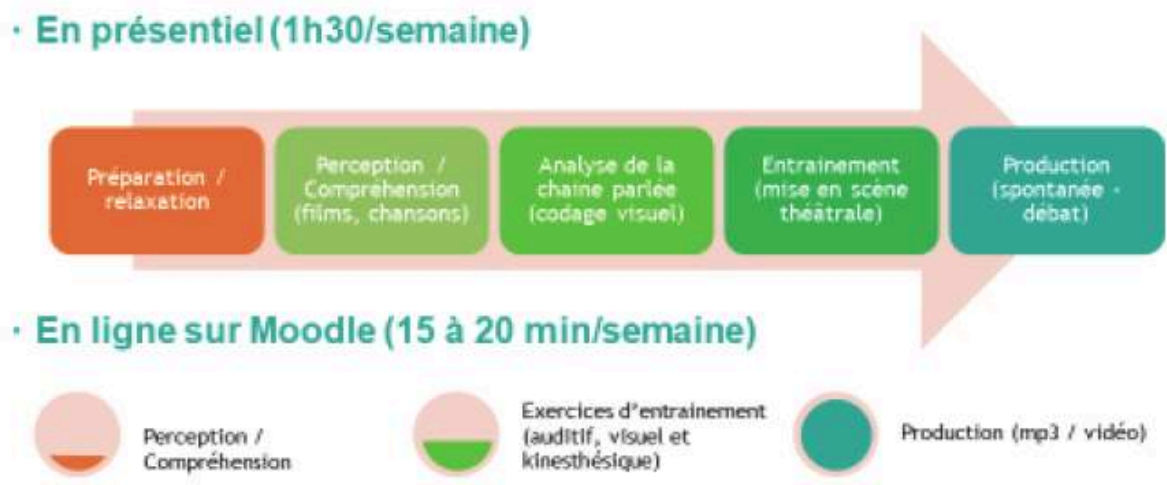

Figure 2 : scénarisation pédagogique du dispositif hybride étudié

- Une première phase concerne la préparation à la production par un massage de l'appareil phonatoire et par des exercices de type virelangues pour l'échauffement.

- La deuxième phase est la phase de perception du rythme par l'activité de compréhension orale des extraits de films: cette phase permet d'être exposé aux composantes vidéo et audio du matériau filmique. 
- La troisième étape concerne le codage rythmique : il est demandé à l'apprenant de coder les groupes rythmiques, les accentuations, les intonations et les liaisons/ enchaînements. Nous y reviendrons plus précisément dans la partie concernant le codage rythmique. Cette phase permet de développer les stratégies d'apprentissage visuelles du rythme.

- La quatrième phase est la mise en scène théatrale des extraits de films en se fondant sur le codage rythmique et l'extrait filmique en vidéo. Cette phase se réalise en binôme. La mise en scène théâtrale permet une interprétation de la scène avec un entraînement de la production orale et notamment du rythme du français. Il s'agit ainsi de mettre en scène le dialogue en choisissant un des rôles issus de l'extrait filmique pour interpréter le dialogue, le phrasé, le rythme et les émotions d'un personnage. Cette phase permet de développer les stratégies d'apprentissage kinesthésiques du rythme en mettant en avant le lien corps/langage pour ressentir le rythme du français par un jeu d'identité.

- La dernière phase concerne la mise en pratique des apprentissages sur le rythme par la production spontanée en interaction à travers des débats sur des thèmes d'actualité pour travailler le rythme selon un entraînement composé d'une approche auditive, visuelle et kinesthésique et, enfin, une production spontanée.

\section{3. Scénarisation du dispositif à distance}

33 L'articulation des activités en présentiel et à distance se réalise par la mise en place d'activités asynchrones sur une plateforme LMS Moodle entre chaque séance en présentiel. Voici la capture d'écran de la séance 4 dédiée à l'exploration spatiale sur Mars sur Moodle :

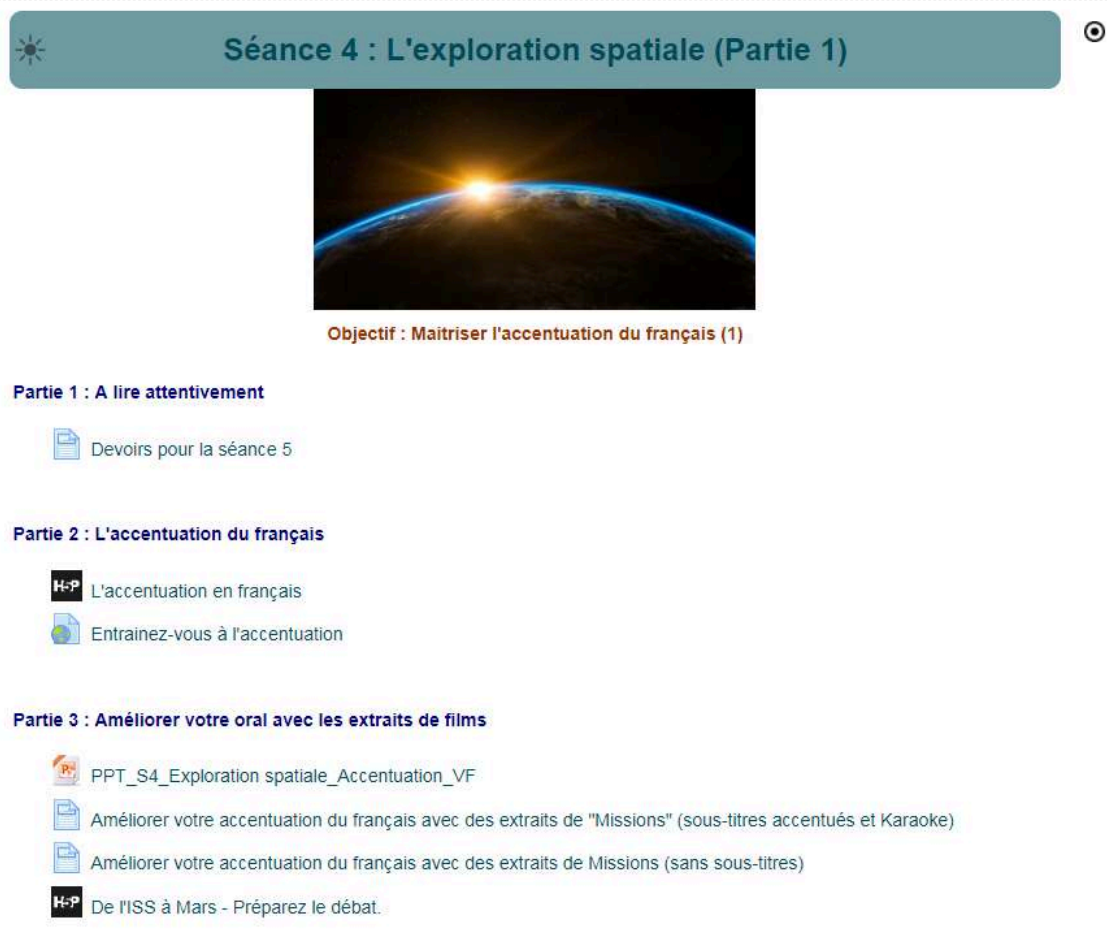

Figure 3 : capture d'écran de la séance 4 du dispositif pédagogique dédiée à l'exploration spatiale 
Vingt minutes d'activités en ligne sont réparties selon trois étapes :

- une première étape de perception/compréhension d'extraits de films avec pour objectif la compréhension orale fine et détaillée d'un extrait de film court de deux minutes maximum, permettant à l'apprenant de percevoir le rythme des personnages. Ces extraits de films sont mis en relation avec des vidéos détaillant théoriquement un des aspects du rythme (accentuation, groupe rythmiques, etc.) sur l'outil H5P (permettant la réalisation de vidéos interactives avec sommaire, signets, quiz, questions, insertion de liens, vidéos, images, sons). Ces vidéos permettent de faire le lien avec les apprentissages en présentiel sur les différentes parties du rythme, permettant ainsi à l'apprenant de revoir à son rythme comment fonctionne le rythme.

- une seconde étape d'entrainement avec le codage visuel des extraits filmiques (des groupes rythmiques, de l'accentuation et de l'intonation - nous reviendrons sur le codage dans la partie suivante), puis la réalisation d'un enregistrement audio ou vidéo au choix de l'apprenant avec une interprétation d'un des rôles du film avec trois supports visuels. La réalisation du codage s'effectue sur le support PowerPoint utilisé en présentiel. Cela permet d'utiliser le même support pour s'entraîner de nouveau sur le même extrait filmique vu en classe de langue. Les corrections du codage visuel sont sur le même PowerPoint en fin d'exercice. Il s'agit ainsi d'un exercice autonome avec une auto-correction. Un exemple du codage visuel sur le PowerPoint sera analysé dans la partie suivante.

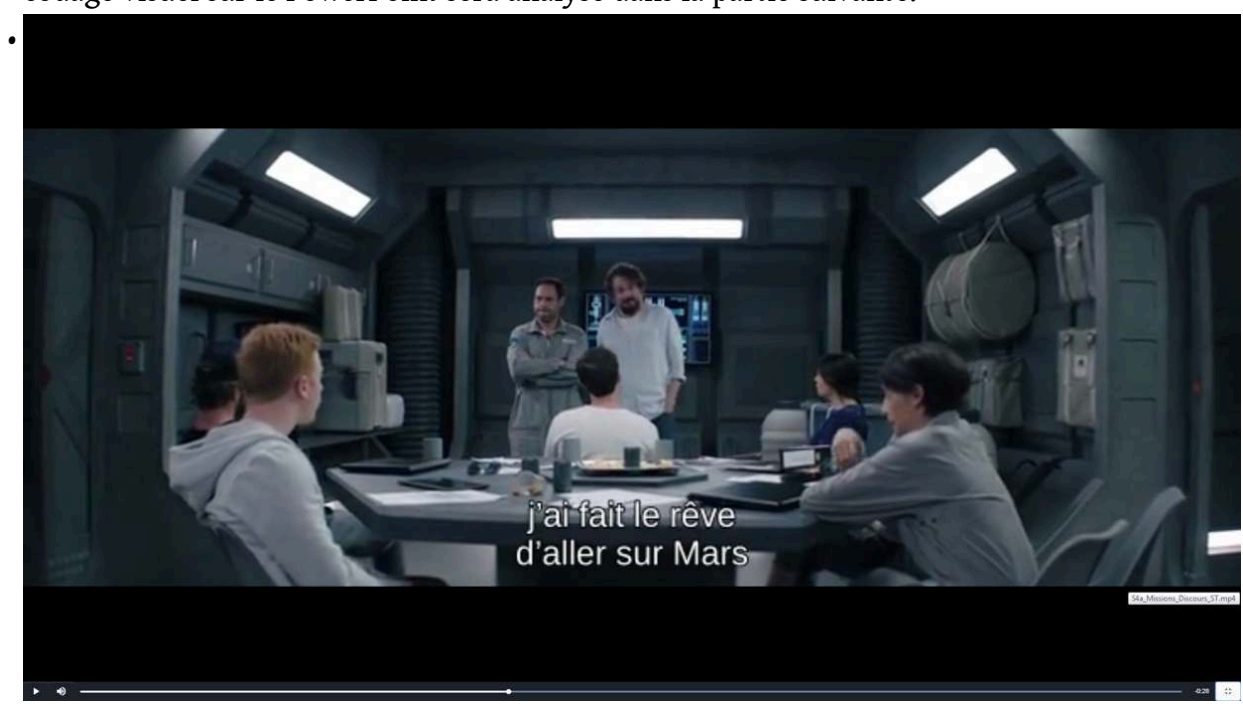

Les trois supports visuels sont trois extraits de films identiques mais ayant des sous-titrages différents : le premier support filmique comporte un sous-titrage intralinguistique (soit en français, la langue cible), comme on peut le voir ici dans la Figure 4. Ce sous-titrage permet à l'apprenant de percevoir à la fois de façon auditive et visuelle les dialogues des personnages. Figure 4 : capture d'écran de la vidéo avec sous-titrage intralinguistique du cours de FLE C1 lors de la séance 4, issue d'un extrait de la série télévisée Missions (2017) d'Henri Debeurme, Julien Lacombe avec Hélène Viviès, Clément Auber (épisode 1)

- Le deuxième support filmique est le même extrait de film avec le codage visuel des accentuations en fin de groupe rythmique en rouge pour mettre en évidence les proéminences en allongeant la durée des syllabes, en donnant plus de force à la syllabe et plus de hauteur par rapport aux syllabes précédentes. Les groupes rythmiques sont marqués par les coupures dans les affichages des sous-titres à l'écran. Nous pouvons le voir dans la Figure 5, où le mot « Mars » est en fin de groupe rythmique et est donc accentué. L'objectif est d'apporter une mémorisation par couleurs et par symboles des différentes composantes du rythme. 


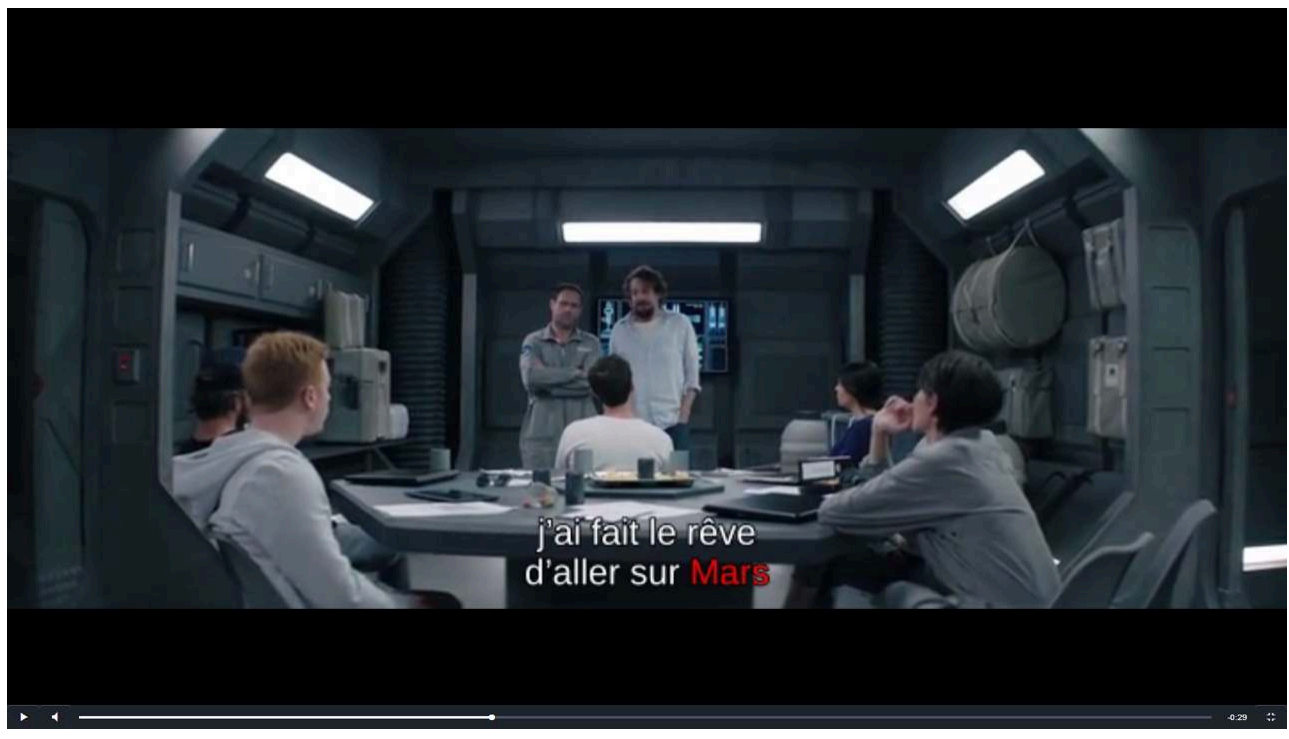

Figure 5 : capture d'écran de la vidéo avec sous-titrage intralinguistique et codage visuel du cours de FLE C1 lors de la séance 4, issue d'un extrait de la série télévisée Missions (2017) d'Henri Debeurme, Julien Lacombe avec Hélène Viviès, Clément Auber (épisode 1)

- Le troisième support filmique à disposition des apprenants est le même extrait de film mais avec un sous-titrage défilant en mode karaoké permettant ainsi de voir chaque syllabe dite par le personnage se colorer en bleu et donc permettant à l'apprenant de s'entraîner sur l'interprétation de la scène en jouant le rôle d'un des personnages. Nous pouvons voir un exemple dans la Figure 6. Les trois étapes correspondent aux trois étapes d'appropriation du rythme: une première étape permettant une redondance des informations auditives et visuelles pour une meilleure mémorisation, puis une mise en relief des paramètres rythmiques tels que l'accentuation et la démarcation des groupes rythmiques pour ancrer les informations à long terme et, enfin, une mise en relief en temps réel des syllabes prononcées par le personnage. Après s'être entraîné sur ces trois vidéos, l'apprenant choisit un des personnages et réalise un enregistrement audio ou vidéo de sa performance en suivant la vidéo de l'extrait du film. Il s'agit pour lui de prendre en compte non seulement le rythme du personnage mais aussi ses émotions marquées dans les paramètres prosodiques. 


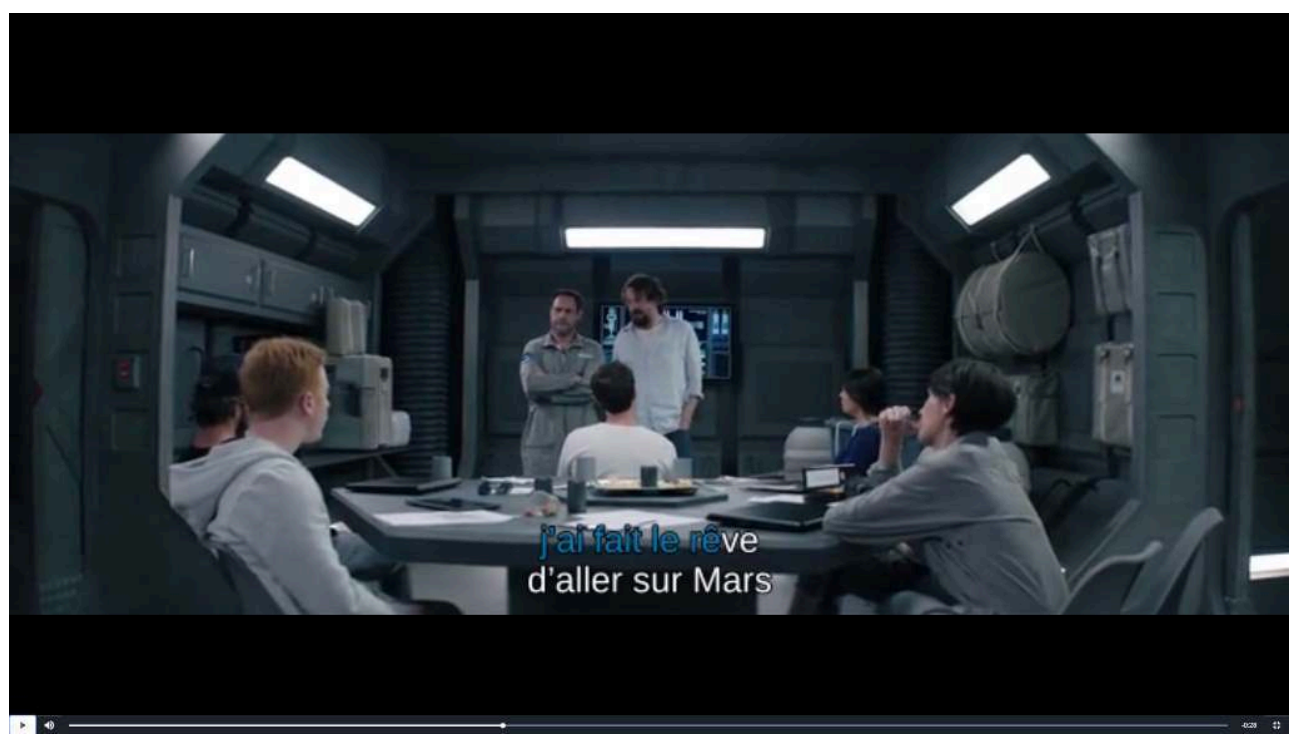

Figure 6 : capture d'écran de la vidéo avec sous-titrage intralinguistique en mode karaoké du cours de FLE C1 lors de la séance 4, issue d'un extrait de la série télévisée Missions (2017) d'Henri Debeurme, Julien Lacombe avec Hélène Viviès, Clément Auber (épisode 1)

- Enfin, une troisième étape avec la réalisation d'un enregistrement audio sur une production spontanée par un débat sur un forum audio: les échanges sont réalisés non pas à l'écrit, comme le serait un forum classique, mais par échanges audio. Les débats, ont pour thème des sujets d'actualités en relation avec les thèmes des extraits de films choisis, l'objectif étant de mettre en pratique tous les éléments appris lors de l'entraînement par un transfert des acquis sur le rythme à travers une production spontanée en interaction asynchrone.

L'articulation entre les séances en présentiel et les activités à distance mettent en relation la flexibilité inhérente du dispositif (Nissen, 2019), où l'apprenant réalise les activités à son rythme en fonction de ses objectifs en matière de prononciation, la variation temporelle avec des activités en synchrone en présentiel et en asynchrone à distance et, enfin, le lien entre les apprentissages en présentiel et à distance. La variation temporelle est une composante des dispositifs hybrides.

\section{4. Corpus et méthodologie d'analyse des données recueillies}

Notre corpus est composé de la scénarisation pédagogique de ce système de codage rythmique au sein d'un dispositif hybride de douze séances de deux heures en présentiel sur douze semaines entrecoupé de douze séances de vingt minutes d'activités en ligne sur la plateforme LMS Moodle. L'objectif pédagogique étant l'appropriation de la prononciation du français, nous avons mis en place une approche multisensorielle alliant la mise en scène théâtrale (mémorisation kinesthésique), d'extraits filmiques (mémorisation sensorielle auditive) et de chansons prenant appui sur le système de codage rythmique mentionné (mémorisation sensorielle visuelle).

Dans notre article, nous nous focaliserons spécifiquement sur la complémentarité de la perception visuelle et auditive du rythme par notre codage rythmique d'extraits de films en tant que genre littéraire. Outre la scénarisation pédagogique, notre corpus est constitué de trois questionnaires (début, mi et fin de formation $(\mathrm{N}=23)$ ayant pour objectif l'appréciation et l'utilité du codage rythmique visuel mis en relation avec six entretiens semi-directifs réalisés avec les apprenants concernant la mise en place de 
stratégies d'apprentissage multisensorielles. L'analyse de ces données fera l'objet d'une prochaine publication.

\section{Vers un codage visuel du rythme}

Les codages visuels du rythme ou encore représentations visuelles du rythme existent depuis les années 1950-1960, notamment dans le domaine de la phonétique acoustique pour une représentation des différents aspects de la prosodie du français. Avant de proposer notre propre codage visuel du rythme à partir de scénario cinématographique, il convient de revenir rapidement sur la revue de littérature sur les représentations visuelles existantes.

Nous savons que la théorie de l'image s'appuie sur la représentation sémiotique (Jacquinot, 1985 ; 2012) et la théorie du double codage (Fraisse, 1975 ; Lieury, 1995) avec un codage visuel des phrases. Les travaux en psychologie cognitive montrent que le traitement des images, du son et d'un codage spécifique permet de générer une image stockée sous forme « iconique » et sous forme « verbale » (Fraisse, 1975 ; Barthes, 1964). Ainsi, chaque image renferme un "message linguistique ", un "message iconique codé » et un "message iconique non codé ». La mémorisation serait améliorée par les deux types de stimuli, soit un stimulus auditif et visuel ${ }^{4}$ (Jamet, 1998).

\section{1. Représentations visuelles de la prosodie}

Du point de vue didactique, les représentations visuelles du système prosodique du français doivent être codées, comme en témoignent les travaux de Di Cristo (2016) dont est issu la Figure 7 :

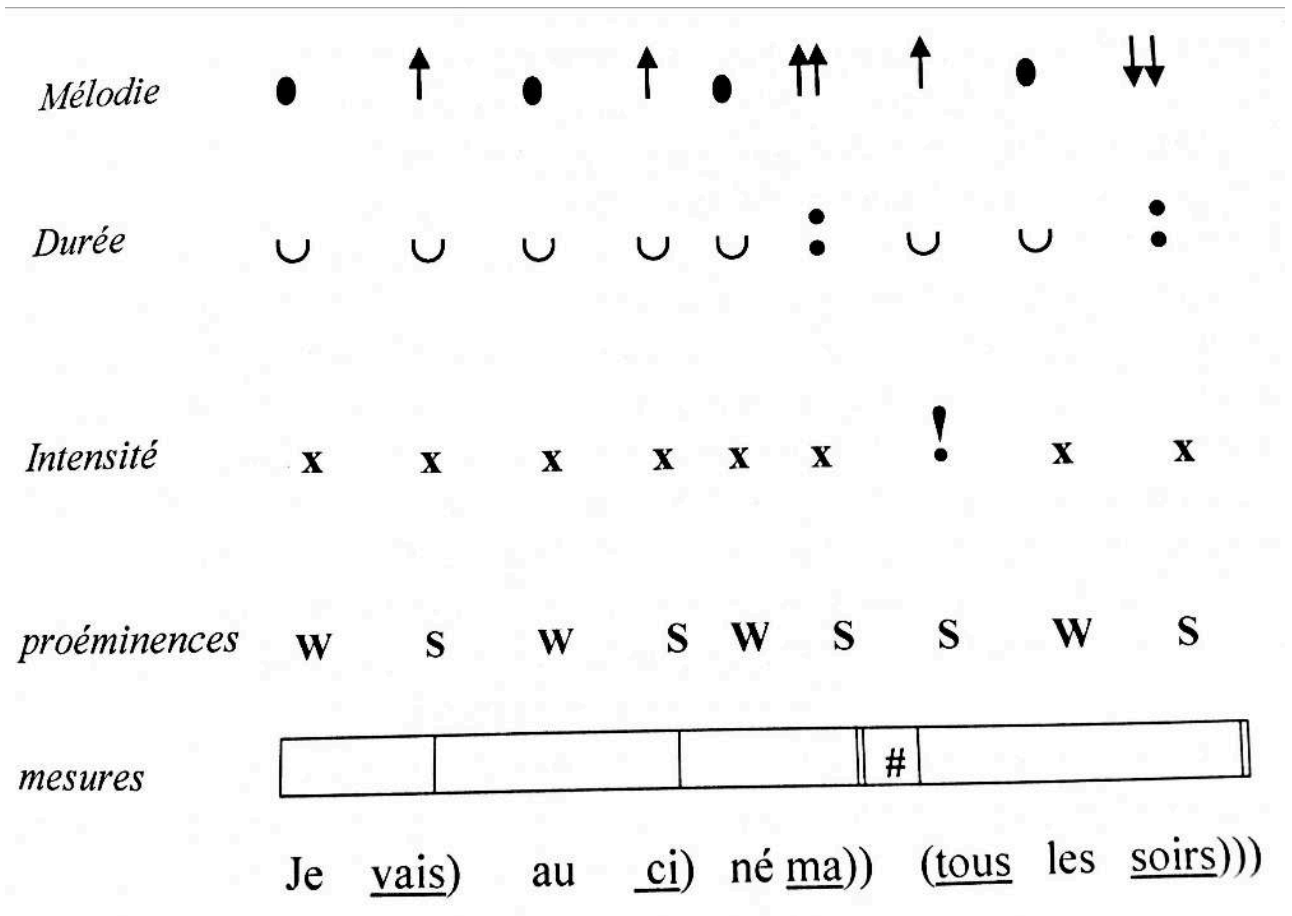

Figure 7 : extrait d'un exemple de représentation visuelle des paramètres prosodiques de l'énoncé « Je vais au cinéma tous les soirs », issu de Di Cristo $(2016: 67)$ 
41 Mais il faut également des marquages automatisés comme systèmes de représentations et de notations à des fins pédagogiques. Les travaux de Herment (2018) montrent que la transcription tonétique interlinéaire permet notamment de marquer les proéminences par l'utilisation des points de plusieurs tailles différentes. Cette représentation est plus souvent utilisée en anglais notamment par la présence des « tones » et « tonicity ».

42 Certains logiciels permettent de réaliser une représentation automatique de plusieurs éléments de la prosodie : par exemple, le logiciel de traitement de la parole PRAAT crée une courbe mélodique en bleu. Le logiciel WinPitch de Philippe Martin permet de réaliser des analyses acoustiques mais possède également une application pédagogique par la visualisation en temps réel de la courbe mélodique et par une comparaison possible avec un modèle (avec une modification de sa propre performance pour correspondre au modèle), l'idée étant de travailler à la fois sur la perception et la production de la prosodie en temps réel.

43 Ces logiciels permettent de réaliser des annotations automatiques utilisées en phonétique acoustique et peu en recherche didactique ou en pratique de classe. Une formation de l'enseignant est donc indispensable avant son utilisation.

44 Ainsi, nous avons voulu utiliser un support pédagogique tel que le scénario cinématographique, accessible à tous les enseignants mais aussi à tous les étudiants : il s'agit de développer un système simple et peu technique pour les enseignants non formés notamment à la phonétique.

45 Nous pouvons désormais traiter de la méthodologie d'usage des scénarios de films avec notre codage rythmique.

\section{2. L'analyse pré-pédagogique des scénarios de films}

La sémiotique de l'image permet de comprendre qu'interpréter des images est un processus analogique (Bourrissoux et Pelpel, 1992). Jacquinot $(1985$; 2012) affirme que la lecture des supports audiovisuels n'est jamais passive, c'est une " activité psychique intense faite de choix, de mise en relation des éléments de l'image entre eux " (Jacquinot, 1985:26). Les facteurs affectifs semblent avoir un impact sur les apprenants.

47 Le choix des extraits de scénarios relève bien entendu d'une analyse pré-pédagogique des différents supports en fonction des objectifs et du niveau des apprenants. Prendre un extrait court (1 à 2 min maximum) permet de se focaliser sur les caractéristiques rythmiques de façon plus affinée. Comment choisir les extraits de films? En fonction des objectifs d'apprentissage des apprenants, nous avons choisis des extraits comprenant des formats de discours, à savoir des extraits filmiques comportant des dialogues constitués d'unités communicationnelles ou d'unités de sens (groupes rythmiques) pour développer les compétences interactionnelles des apprenants. On peut aussi prendre des extraits de discours journalistiques pour développer les compétences d'expression orale argumentées de futurs journalistes.

L'analyse pré-pédagogique de l'extrait de film (Decock, 1970; Compte, 1993) va permettre également de choisir le film en fonction des thèmes du cours de langues. En fonction du niveau des apprenants, on peut choisir des extraits de films en fonction de différents niveaux de difficultés sur le plan du rythme (par exemple, les groupes rythmiques et l'accentuation pour les niveaux A1 à B2, le schwa, les liaisons et les 
enchaînements pour les niveaux $\mathrm{B} 1$ à $\mathrm{C} 1$, les intonations pour les niveaux $\mathrm{A} 1$ à $\mathrm{C} 1$ ). Ils peuvent être sélectionnés en fonction du niveau de difficulté de la perception de la variation diaphasique (registres de langue : familier, courant, standard, académique, soutenu), de la maîtrise du lexique ou de la morphosyntaxe orale (Blanche-Benveniste, 1987). Nous choisirons pour l'exposé la ressource audio-visuelle en fonction de la pertinence du contenu mais aussi en fonction des indices accentuels, c'est-à-dire un extrait pour illustrer un seul phénomène qu'il soit segmental ou suprasegmental afin de ne pas créer de surcharge cognitive. Il s'agit à présent de voir comment rendre saillants ces traits rythmiques.

\section{3. Méthodologie de l'élaboration du codage rythmique : coder les paramètres prosodiques de mélodie, de durée et d'intensité} l'appropriation
universitaire :

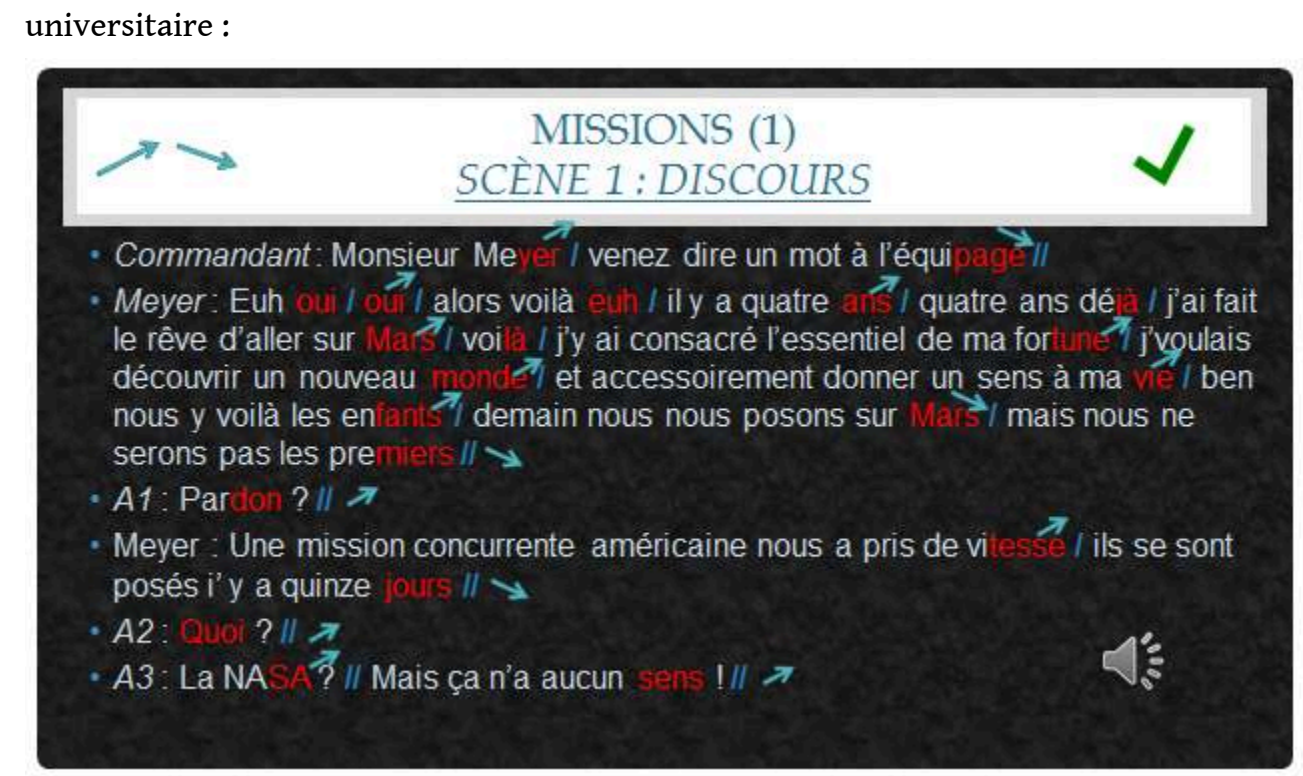

Figure 8 : capture d'écran du PowerPoint utilisé en cours de FLE C1 lors de la séance 4 avec un extrait du codage visuel du rythme, issue d'un extrait de la série télévisée Missions (2017) d'Henri Debeurme, Julien Lacombe avec Hélène Viviès, Clément Auber (épisode 1)

51 Le cours de FLE était basé sur des thèmes d'actualités en relation avec les domaines d'études des apprenants. Le thème de l'exploration de «Mars » étant un thème d'actualité récurrent en 2018, il s'avérait judicieux de l'exploiter (série télévisée française sur l'exploration de Mars): l'objectif phonétique était l'appropriation des syllabes accentuées en fin de groupe rythmique. Pour atteindre notre objectif, un dialogue entre différents membres de l'équipage rendait compte de l'interaction orale, puis la présence d'un dialogue plus long offrait la présence de plusieurs groupes rythmiques et donc de plusieurs syllabes accentuées.

On peut observer ici :

- Les barres obliques (ici en bleu) permettent de délimiter les groupes rythmiques qui correspondent aux groupes de sens ; 
- L'apprenant peut les percevoir par la combinaison des facteurs temporels mais également des facteurs non temporels, comme la montée de continuation terminant un groupe rythmique non final en français : c'est cette montée qui, sans forcément être suivie d'une pause, peut marquer une frontière finale de groupe rythmique en français ;

- Une barre oblique en bleu indique une courte pause (au sein d'une idée) ;

- Deux barres obliques indiquent une pause plus longue (entre deux idées distinctes, entre deux phrases);

- Les syllabes en rouge permettent de mettre en valeur les syllabes accentuées qui correspondent aux syllabes qui sont plus longues, plus fortes et plus hautes que les syllabes non accentuées. Elles se trouvent en fin de groupe rythmique;

- Les syllabes non accentuées sont ici notées en blanc ;

- La couleur rouge est plus forte au niveau visuel et plus attirante, et permet à l'apprenant de visualiser rapidement les syllabes à accentuer ;

- En plus du rythme, les flèches montantes indiquent les mouvements de la voix par une intonation montante et les flèches descendantes représentent une intonation descendante ;

-L'intonation, élément de la prosodie, est intimement liée au rythme car l'intonation est une marque distinctive notamment de l'émotion du locuteur.

53 C'est ainsi que nous avons pu coder la langue parlée telle que reproduite dans les films. Nous l'avons systématiquement couplé à l'extrait audio et vidéo du film afin de développer l'appropriation auditive et visuelle du rythme de la langue étrangère.

\section{4. Scénarisation pédagogique du codage rythmique}

54 À présent nous pourrons aborder l'exploitation pédagogique de ce système de codage rythmique en représentant un extrait de notre scénarisation pédagogique d'un cours hybride (alternant des moments en présentiel et des moments à distance) intitulé " “Améliorer sa prononciation du français" DUEF 2-Niveau C1» à l'Université Sorbonne Nouvelle-Paris 3.

L'expérimentation de ce codage rythmique a été réalisée en deux temps : un premier pendant le cours en présentiel à raison de deux heures par semaine à l'aide d'une transcription du scénario cinématographique de la série Missions et l'autre durant les activités à distance sur Moodle à raison de vingt minutes par semaine entre chaque séance en présentiel.

\section{4. 1. Au sein d'un cours hybride " "Améliorer sa prononciation du français" DUEF 2 - Niveau $\mathrm{C} 1 »$ : cours en présentiel}

Le codage rythmique est réalisé par les apprenants. La première étape des activités consiste en la transcription d'un extrait de films courts (2-3 minutes). Cette étape peut être réalisée par les apprenants permettant ainsi une meilleure perception et compréhension de la chaîne parlée en temps réel.

Dans un deuxième temps, le codage est introduit de façon séquentielle avec, d'abord, le travail sur les groupes rythmiques, puis les accentuations, enfin des intonations. Au fur et à mesure du semestre, le codage devient automatique.

Ce codage rythmique a pour objectif de favoriser l'appropriation visuelle. La mise en scène théâtrale de l'extrait du film doit développer à la fois l'appropriation visuelle et kinesthésique du rythme. Il s'agit ainsi de faire rejouer la scène de l'extrait du scénario 
du film aux apprenants en classe de langue. En effet, selon Jousse (1981), l'ancrage corporel permet à l'apprenant de faciliter l'entrée dans l'apprentissage d'une langue et notamment du rythme de la langue. L'activité de phonation et de réception est une activité corporelle qui mobilise les organes de la parole mais aussi le cerveau pour les traitements de la mélodie et des sons qui composent la phonation. En prenant la langue non plus comme un jeu intellectuel mais comme un jeu corporel, l'apprenant explore les différentes facettes de son propre corps et des possibilités qui lui sont offertes. L'appropriation de la langue devient donc multisensorielle en ce qu'elle engage le corps, ses émotions et ses sensations. Le jeu scénique, appelé également « mise en scène théâtrale ", permettrait de mettre à profit l'expression corporelle pour ressentir physiquement le rythme de la langue. La variation temporelle dans le processus d'appropriation du rythme est aussi une variable importante dans l'appropriation.

Nous allons maintenant montrer comment ce codage rythmique a pu s'opérer dans un cours avec un extrait filmique mis en ligne sur Moodle dans les activités à distance.

\section{4. 2. Cours hybride " "Améliorer sa prononciation du français" DUEF 2 - Niveau $\mathrm{C} 1 »$ : activités à distance}

Entre chaque séance en présentiel de deux heures, nous avons vingt minutes d'activités en ligne sur la plateforme LMS Moodle, où les apprenants ont la possibilité de visionner plusieurs fois les vidéos des extraits filmiques visionnées en présentiel avec un système de sous-titrage reproduisant le codage visuel rythmique ainsi élaboré.

61 Trois vidéos du même extrait filmique sont proposées à l'apprenant : une première avec les sous-titres intralinguistiques classiques en blanc, une deuxième avec un sous-titrage intralinguistique avec les accentuations des syllabes en rouge et des groupes rythmiques (voir Figure 9) et, enfin, une troisième vidéo avec un sous-titrage de type Karaoké où les sous-titres se colorent en bleu au fur et à mesure que les personnages s'expriment.

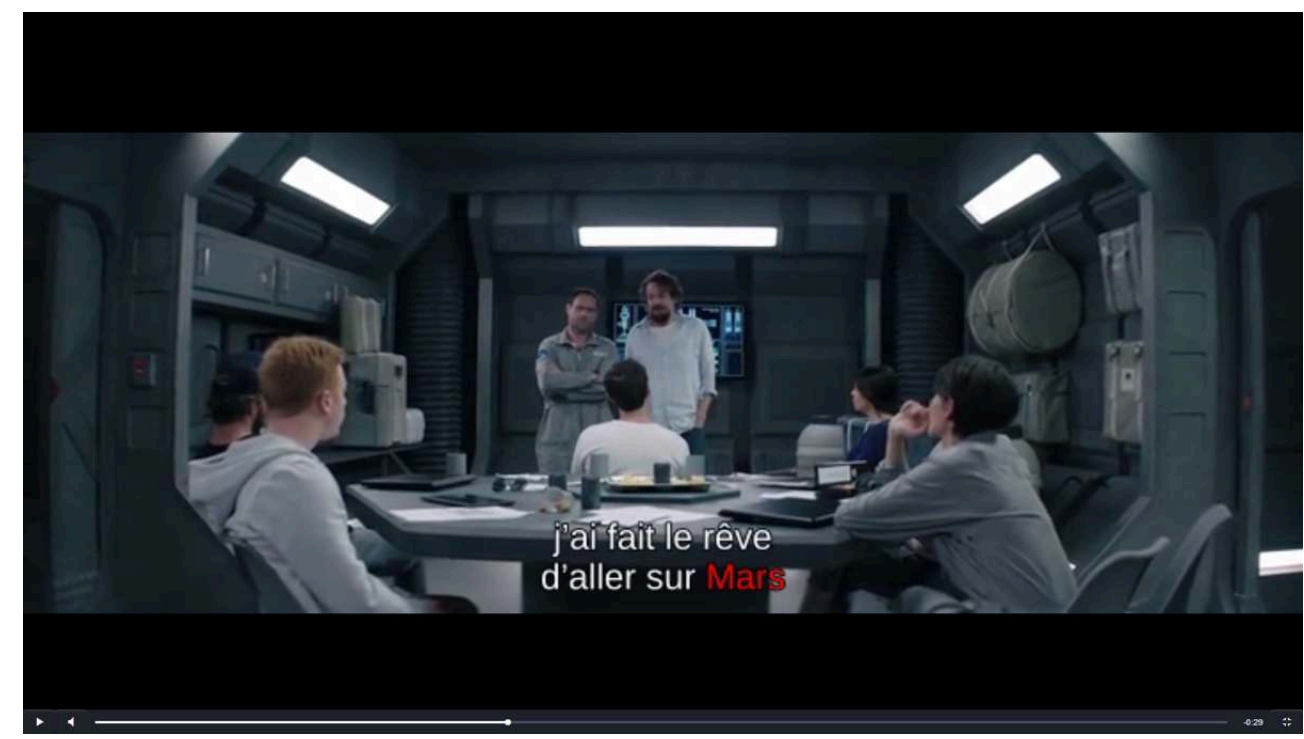

Figure 9 : capture d'écran de la vidéo avec sous-titrage intralinguistique du cours de FLE C1 lors de la séance 4 avec un extrait du codage visuel du rythme, issue d'un extrait de la série Missions (2017) d'Henri Debeurme, Julien Lacombe avec Hélène Viviès, Clément Auber (épisode 1) 

trois points suivants :

1. Une hypothèse forte semble se dessiner concernant la variation temporelle de la réalisation du codage rythmique : elle aurait des impacts sur la mémorisation sensorielle. En effet, lors de la séance en présentiel, nous réalisons le codage rythmique en synchrone, puis lors des activités à distance, le codage rythmique est présenté en asynchrone à travers des vidéos avec sous-titrage présentant les groupes et l'accentuation. Cet écart temporel permet un meilleur traitement des images et du son avec un encodage fondé sur le double codage visuel et auditif (Cordier et Gaonac'h, 2010).

2. Le double codage de l'information (auditif et visuel) permet un meilleur traitement de l'information et donc une meilleure mémorisation du rythme par le traitement sensoriel auditif et visuel. On constate cependant que le double traitement visuel et auditif demande un temps d'adaptation: on peut formuler l'hypothèse d'une surcharge cognitive qui peut survenir chez les apprenants. Il y a pour certains apprenants trop d'informations, notamment de couleurs ne leur permettant pas de traiter en même temps les éléments prosodiques et le sens donné au codage (couleurs différentes). Cette dimension mérite une exploration plus approfondie.

3. Il semble enfin que la mémorisation du rythme se réalise par un traitement séquentiel des informations avec des stimuli sensoriels tel qu'envisagé par les travaux en cognition : il reste à savoir comment organiser et ajuster ce traitement multisensoriel du rythme (Cordier et Gaonac'h, 2010).

On peut dire que les stratégies d'apprentissage visuelles et auditives (par les extraits de films) développées par les apprenants lors de la réalisation d'un codage visuel d'extraits audio de scénarios filmiques sont difficiles à tracer et à répertorier : reste à s'interroger 
sur la méthodologie expérimentale pour saisir leur efficacité. La poursuite de cette recherche devrait pouvoir rendre compte de l'efficacité de l'appropriation de la prosodie (avec un accent particulièrement mis sur l'appropriation multisensorielle du rythme) par le développement de stratégies d'apprentissage multisensorielle (notamment visuelles en ce qui concerne le codage rythmique visuel et auditif par la compréhension orale d'extraits de scénarios filmiques).

Enfin, si ce dispositif a été élaboré en hybride avec une alternance entre des moments en présentiel et des moments à distance sur une plateforme LMS Moodle, il reste à déceler dans quelle situation d'enseignement/apprentissage cette mise en œuvre est pertinente ou non.

Si l'utilisation de scénarios filmiques en tant que texte littéraire permet aux apprenants de s'approprier un schéma narratif, des personnages avec une mise en mouvance des émotions et un cadre socio-culturel ancré dans un contexte linguistique, il permet de déployer la production orale (jouer un rôle par rapport à un acteur ou à un comédien), d'imiter ses gestes, son phrasé et ses émotions comme reflet du rythme de la langue étrangère. Pour terminer on peut pointer que ce modèle peut aider l'apprenant à prendre plus de risque notamment sur le plan de la prononciation puisqu'il offre une distanciation par rapport à sa propre identité ; dans un contexte plus traditionnel, l'apprenant n'oserait peut-être pas jouer "son rôle». De toute évidence la combinatoire - scénario filmique et codage rythmique - est une activité métalangagière et métaphonologique intéressante et une ouverture consciente et raisonnée au rythme du français à la fois aux niveaux auditif et visuel par différentes mises en place de stratégies d'apprentissage (sous-titrage intralinguistique mettant en valeur les syllabes accentuées et les groupes rythmiques notamment). Ces dispositifs sont destinés à prendre de l'ampleur à l'heure du numérique dans l'enseignement/apprentissage des langues.

\section{BIBLIOGRAPHIE}

ABERCROMBIE D., 1964, « A phonetician's view of verse structure », Linguistics, vol. 2, nº 6, p. 5-13.

ANDERSON-HSIEH J., 1992, « Using electronic visual feedback to teach suprasegmentals », System, vol. $20, n^{\circ} 1$, p. 51-62.

ATLAN J., 1995, « Stratégies d'apprentissage d'une langue étrangère et traitement pédagogique : aperçu d'une méthodologie de recherche ", ASp [En ligne], $\mathrm{n}^{\circ}$ 7-10, mis en ligne le 19 novembre 2013. Disponible sur : <http://journals.openedition.org/asp/3985>.

BANDURA A., 2003, Auto-efficacité: le sentiment d'efficacité personnelle, Paris, De Boeck Université, coll. « Ouvertures Psy ».

BARTHES R., 1964, « Rhétorique de l'image », Communications, vol. 4, nº 1, p. 40-51.

BOURRISSOUX J. L. et PELPEL P., 1992, Enseigner avec l'audiovisuel, Paris, Les Éditions d'Organisation.

COMPTE C., 1993, La vidéo en classe de langue, Paris, Hachette, coll. « $\mathrm{f} /$ autoformation ». 
DECOCK J., 1970, « L'Utilisation du film commercial dans l'enseignement des langues », The French Review, vol. 43, $\mathrm{n}^{\circ}$ 3, p. 467-473.

DEWEY J., 1916, Democracy and education : An introduction to the philosophy of education, New-York, Macmillan.

DI CRISTO A. et HIRST D., 1993, « Rythme syllabique, rythme mélodique et représentation hiérarchique de la prosodie du français ", Travaux de l'Institut de Phonétique d'Aix, n 15, p. 9-24.

DI CRISTO A., 1999, «Vers une modélisation de l'accentuation du français : première partie », Journal of French language studies, vol. 9, nº 2, p. 143-179.

-, 2004, « La prosodie au carrefour de la phonétique, de la phonologie et de l'articulation formesfonctions ", Travaux Interdisciplinaires du Laboratoire Parole et Langage d'Aix-en-Provence (TIPA), $\mathrm{n}^{\circ}$ 23, p. 67-211.

-, 2016, Les musiques du français parlé: Essais sur l'accentuation, la métrique, le rythme, le phrasé prosodique et l'intonation du français contemporain, vol. 1, Berlin/Boston, Walter de Gruyter GmbH \& Co KG, coll. «Études de linguistique française - Collection ILF ».

EUSTACHE F. et DESGRANGES B., 1999, « Vers un modèle unifié de la mémoire », Cerveau \& Psycho, nº 28. FÓNAGY I., 1980, « L'accent français : accent probabilitaire (dynamique d'un changement prosodique) ", Studia Phonetica Montréal, nº 15, p. 123-233.

FRAISSE P., 1975, « Influence du codage visuel de phrases sur leur mémorisation à court terme », L'Année psychologique, vol. 75, n² 2, p. 409-416.

GAONAC'H D. et CORDIER F., 2010, Apprentissage et mémoire, Paris, Armand Colin, coll. « 128 ».

GUIMBRETIÈRE É., 1994, Phonétique et enseignement de l'oral, Paris, Didier Hatier.

HERMENT S., 2018, « Apprentissage et enseignement de la prosodie : l'importance de la visualisation », Revue française de linguistique appliquée, vol. 23, n 1, p. 73-88.

HAYES B., 1995, Metrical stress theory : Principles and case studies, Chicago, University of Chicago Press.

HUART R., 2002, La grammaire orale de l'anglais, Paris, Ophrys.

JACQUINOT G., 1985, L'école devant les écrans, Paris, Éditions ESF, coll. « Science de l'éducation ».

-, 2012 [1977], Image et pédagogie, Paris, Éditions des archives contemporaines.

JAMET E., 1998, «L'influence des formats de présentation sur la mémorisation », Revue de Psychologie de l'Éducation, $\mathrm{n}^{\circ}$ 1, p. 9-35.

JOUSSE M., 1981, Le style oral rythmique et mnémotechnique chez les verbo-moteurs, Paris, Fondation Marcel Jousse, vol. 2.

KAGLIK A. et BOULA DE MAREÜIL P., 2009, « Perception d'un accent étranger et part de la prosodie selon l'âge de première exposition à la L2 : transfert ou phénomène universel en acquisition ", $6^{e s}$ Journées d'Études Linguistiques, p. 7-13.

KRASHEN S., 1992, « The Input Hypothesis : An Update », Linguistics and language pedagogy : The state of the art, p. 409-431.

LANCIEN T., 1986, Le document vidéo dans la classe de langues, Paris, CLE international, coll. « Techniques de classe». 
-, 2004, De la vidéo à Internet : 80 activités thématiques, Paris, Hachette FLE, coll. « Pratiques de classe ».

LAURET B., 2007, Enseigner la prononciation du français : questions et outils, Paris, Hachette, coll. «f $»$.

LÉON P. R., 2011, Phonétisme et prononciations du français, $6^{\mathrm{e}}$ éd., Paris, Armand Colin, coll. « Cursus ».

LIBERMAN M. et PRINCE A., 1977, « On stress and linguistic rhythm », Linguistic inquiry, vol. 8, $\mathrm{n}^{\circ}$ 2,

p. 249-336.

LIEURY A., 1995, « Mémoire des images et double codage », L'année psychologique, vol. 95, nº 4, p. 661-673.

MAURY C., 2012, "L'intégration du cinéma hollywoodien en classe de langue : réflexions sur quelques points de convergence disciplinaires ", Recherche et pratiques pédagogiques en langues de spécialité. Cahiers de l'Apliut, vol. 31, nº 2, p. 26-43.

MARTIN P., 2012, «Intonation, rythme et eurythmie de locutions et proverbes français ", in J.C. ANSCOMBRE, B. DARBORD et A. ODDO (dir.), La parole exemplaire, introduction à une étude linguistique des proverbes, Paris, Armand Colin, p. 159-169.

MAYER R. E., 1997, « Multimedia learning : are we asking the right questions », Educational Psychologist, vol. 32, nº 1, p. 1-19.

MAYER R. E. et MORENO R., 1998, « A split attention effect in multimedia learning : Evidence for dual processing systems in working memory ", Journal of Educational Psychology, vol. 90, $\mathrm{n}^{\circ} 2, \mathrm{p}$. 312-320.

MESCHONNIC H., 1982, Critique du rythme. Anthropologie historique du langage, Lagrasse, Verdier. MOREL M. A. et DANON-BOILEAU L., 1998, Grammaire de l'intonation l'exemple du français, Paris, Ophrys. MYERS M. J., 2004, Modalités d'apprentissage d'une langue seconde, Bruxelles/Paris, De Boeck supérieur. NARCY-COMBES J.-P., 2005, Didactique des langues et TIC : vers une recherche-action responsable, Paris, Ophrys.

NISSEN E., 2019, Formation hybride en langues. Articuler présentiel et distanciel, Paris, Didier, coll. « Langues \& didactique».

ROSSI M., 1971, « Le seuil de glissando ou seuil de perception des variations tonales pour les sons de la parole ", Phonetica, vol. 23, $\mathrm{n}^{\circ}$ 1, p. 1-33.

-, 1999, L'intonation : le système du français: description et modélisation, Paris, Ophrys.

TORTEL A., 2009, Évaluation qualitative de la prosodie d'apprenants français : apport de paramétrisation prosodiques, thèse de doctorat sous la direction de D. Hirst soutenu à l'Université d'Aix-Marseille Université de Provence. Disponible sur : <https://tel.archives-ouvertes.fr/tel-00455248/ document>.

VERMEESCH A., 2004, «Poétique du scénario », Poétique, vol. 138, nº 2, p. 213-234. Disponible sur : <https://www.cairn.info/revue-poetique-2004-2-page-213.htm>.

WACHS S., 2011, «Tendances actuelles en enseignement de la prononciation du français, langue étrangère (FLE) ", Revista de Lenguas Modernas, $\mathrm{n}^{\circ}$ 14, p. 183-196. Disponible sur : <https:// revistas.ucr.ac.cr/index.php/rlm/article/viewFile/9687/9134>. 


\section{NOTES}

1. La réduction vocalique présente en anglais où la voyelle tendrait à se neutraliser, la syllabation fermée pour l'anglais d'après Léon (2011:135) où l'on constate que $31,8 \%$ des syllabes en anglais sont formées par un type syllabique CVC (Consonne - Voyelle - Consonne), alors que $59,0 \%$ des syllabes en français sont formées par un type syllabique CV (Consonne - Voyelle).

2. En effet, la mémorisation sensorielle, appelée aussi échoïque pour la mémorisation des sons, est une mémoire auditive qui stocke pendant 2 à 3 secondes des informations de types stimuli auditifs. Lorsque l'information est jugée pertinente lors du traitement des sons, ces stimuli sont envoyés vers la mémoire de travail. Celle-ci stocke temporairement les informations durant 16 à 18 secondes environ. Lorsque les 18 secondes sont atteintes, environ $90 \%$ des informations vont être oubliées, sauf si une activité de répétition a été mise en place.

3. Nadia Bacor, 2019, Vers un dispositif hybride pour l'enseignement/apprentissage du rythme de l'anglais et $d u$ français par une approche filmique, théâtrale et musicale, thèse de doctorat en Didactique des Langues, Université Sorbonne Nouvelle-Paris 3, sous la direction de Corinne Weber (Université Sorbonne Nouvelle-Paris 3) et Martine Derivry (Université de Bordeaux).

4. $\mathrm{Si}$ « des illustrations ou des animations sont proposées conjointement à un texte, les résultats sont en faveur d'un texte écouté parallèlement à la présentation des images, plutôt que d'un texte lu (Mousavi, Low et Sweller, 1995 ; Mayer et Moreno, 1998)» (Jamet, $1998: 12$ ).

5. Ce système a été construit en se fondant sur les travaux de phonétique en anglais (Huart, 2002).

\section{RÉSUMÉS}

Dans cette contribution, nous nous proposons d'aborder la perception multisensorielle du rythme du français par la réalisation d'un codage qui s'appuie sur un système de couleurs et de symboles d'extraits filmiques ayant pour objectif l'appropriation du rythme du français. Il s'agira donc d'apporter un éclairage théorique et pédagogique sur l'enseignement/apprentissage du rythme du Français Langue Étrangère (FLE) en classes de langue pour des apprenants de FLE nonspécialistes de langues. Dans un premier temps, nous présenterons les fondements théoriques sur lesquels repose notre approche pédagogique. Ensuite, nous mettrons en pratique le codage rythmique avec des exemples issus d'une expérimentation dans le cadre d'une recherche-action par un dispositif hybride ayant pour objectif l'appropriation de la prononciation du français au niveau $\mathrm{C} 1 \mathrm{du}$ Cadre européen commun de référence pour les langues (CECRL) en contexte universitaire.

In this contribution, we propose to address the multi-sensory learning of the French rhythm by developing a colour coding and symbols of film excerpts in order to acquire French rhythm. The aim will therefore be to provide theoretical and pedagogical insight into the teaching-learning of the French as a Foreign Language (FLE) rhythm in language classes for non-specialist language learners of FLE. First, we will present the theoretical foundations on which our pedagogical approach is based and then we will put into practice rhythmic coding with examples from an experiment as part of an action-research project through a hybrid device aimed at the 
acquisition of French pronunciation at level $\mathrm{C} 1$ of the Common European Framework of Reference for Languages (CEFRL) in a university-level setting.

INDEX

Mots-clés : Prononciation, rythme, perception, auditif, visuel, multi sensoriel, films.

Keywords : Pronunciation, rhythm, perception, auditory, visual, multi-sensory, movies.

\section{AUTEUR}

NADIA BACOR

EA 2288 DILTEC, Université Sorbonne Nouvelle-Paris 3EA 7437 LACES, Université de Bordeaux 